\title{
Effectiveness of a novel oral combination of D-Mannose, pomegranate extract, prebiotics and probiotics in the treatment of acute cystitis in women
}

\author{
Dario Pugliese $^{1}$, Anna Acampora ${ }^{2}$, Angelo Porreca ${ }^{3}$, Luigi Schips ${ }^{4}$, Cindolo Luca ${ }^{1}$ \\ ${ }^{1}$ Department of Urology "Villa Stuart" Private Hospital, Rome, Italy; \\ 2 Institute of Public Health, Catholic University of Sacred Heart, Rome, Italy; \\ ${ }^{3}$ Department of Robotic Urological Surgery, Abano Terme Hospital, Abano Terme, Italy; \\ ${ }^{4}$ Department of Urology, "G. D'Annunzio" University, Chieti, Italy.
}

\begin{abstract}
Summary Objective: Urinary tract infections (UTIs) are defined as the symptomatic presence of pathogens in the urinary tract that are typically diagnosed by microscopy and culture of urine samples. Over the long-term antibiotic courses, alternative prophylactic methods as probiotics, cranberry juices and D-mannose have been introduced for recurrence prevention. The present study aimed to determine whether a new combination of D-Mannose, Pomegranate extract, Prebiotics and Probiotics is effective in modifying symptoms reported by women with acute uncomplicated acute cystitis.

Material and methods: This is a pilot study, performed between September 2018 and November 2018 at the Department of Urology of Villa Stuart Private Hospital. A dose of a new combination of agents was administered twice daily for 5 days and then once a day for 10 days. Together with the compound, forced hydration (> 2 liters/day) has been strongly suggested. Antibiotics were permitted only in case of clinical worsening. Changes in patients' symptoms, the therapeutic effects and changes in quality of life (2oL) were evaluated clinically and through a validated questionnaire, the Acute Cystitis Symptom Score (ACSS) at the first visit (T0), 15 (T1) and 30 (T2) days later.

Results: Thirty-three patients were enrolled in the study (mean age 38,1 \pm 11.2 years) and all completed the treatment protocol. At $T 1$ visit, all symptoms or the majority of symptoms went off in 10 women (30.3\%) and at T2 in 30 women (90.9\%); some symptoms still remained in 16 women (48.5\%) at T1 and in 3 women (9.1\%) at T2; the persistence of all symptoms or the worsening of the condition was observed in 7 patients (21.2\%) at T1 and in none at T2. The mean score reported at all the ACSS sub-scales significantly decreased between baseline and T1 and T2. Typical symptoms decreased from $11.5(10.5-12.6)$ to 4.9 (4.0-5.9) and to 2.7 (2.1-3.3) (p-values < 0.0001); differential symptoms decreased from 3.1 (2.6-3.6) to $0.6(0.3-0.9)$ and to $0.3(0.1-0.5)$ (p-values 0.009 to < 0.0001); QoL mean score also decrease from 7.2 (6.77.7) to 4.0 (3.3-4.6) and to 1.7 (1.2-2.1) (p-values < 0.0001). Six patients required antibiotics and no adverse events were recorded. Conclusions: Our study suggests that the action of the compounds, administered in this new combination, could help in an effective management of symptoms of acute cystitis in women, without antibiotics, in a wide majority of the cases. Lack of microbiological assessment is a clear limitation of the study. Moreover, lack of a control group is another important limitation. Finally, hyperhydration could have been a confounding factor in interpretation of results.
\end{abstract}

KEY WORDS: Urinary tract infection; Cystitis; Female; Fructooligosaccharide; Mannose; Prebiotics; Probiotics; Pomegranate; Symptom score.

Submitted 9 August 2019; Accepted 22 November 2019

\section{INTRODUCTION}

Urinary tract infections (UTIs) are common in general practice, and their diagnosis relies on a combination of characteristic signs and symptoms, urinalysis and a positive urine culture (1). An acute uncomplicated cystitis corresponds to a subset of UTIs including new-onset or recurrent cystitis in non-pregnant women, female patients without relevant anatomical or functional abnormalities of the urinary tract, not related to indwelling urinary catheters, and in the absence of relevant comorbidities such as renal diseases, immunocompromising diseases or diabetes $(2,3)$.

Nowadays, antibiotic therapy represents the treatment of choice, recommended by EAU guidelines, as it is effective towards the rapid symptoms resolution (2).

However, antibiotic usage is related to side effects and increases resistance rates and, consequently, leads to high medical costs, prolonged hospital stays, and increased mortality $(2,4-5)$. Thus, alternative therapies are needed to reduce the risk of antimicrobial resistance development. Probiotics (6), cranberry products (7) and D-mannose (8) have been introduced and studied as a single component or in several combinations (9).

The aim of this pilot study was to investigate whether a new combination of D-mannose, pomegranate extract, prebiotics and probiotics is effective in modifying symptoms reported by women with acute uncomplicated cystitis.

\section{MAterial AND Methods}

This is an uncontrolled experimental pilot study, performed between September 2018 and November 2018 at the Department of Urology of Villa Stuart Private Hospital. All patients provided written informed consent. The trial was conducted according to the Good Clinical Practice guidelines and the declaration of Helsinki (10) and was approved by the local ethics committee.

Female patients admitted at our institution during the recruitment period, complaining of urinary symptom suggestive of UTI were included in the study. Patients were excluded if they presented relevant comorbidities. The exclusion criteria were: pregnancy, antibiotic therapy for UTI within the last month, genital infection, not 
well compensated diabetes mellitus, abnormality of the urinary tract, signs of pyelonephritis (i.e. fever over $38^{\circ} \mathrm{C}$, chills, kidney tenderness by palpation), urine incontinence requiring pads. At the time of inclusion (TO), eligible patients completed the Acute Cystitis Symptom Score (ACSS), a validated questionnaire, specifically developed for self-assessment of acute uncomplicated cystitis (AUC) assessing typical and differential symptoms, quality of life, and changes after therapy in female patients with AUC (11-13). Typical symptoms including frequency, urgency, pain or burning with urination, pain in the suprapubic area, feeling of incomplete bladder emptying and gross hematuria were scored from 0 (not at all) to 3 (severe). Secondly, differential symptoms, such as low back pain, vaginal discharge, urethral discharge or fever and chills were also scored from 0 (not at all) to 3 (severe). In addition, patients recorded how much they felt bothered by their symptoms and how much symptoms impacted on their work/everyday practice and social activities (impairment score, range 0 to 3). Changes from baseline at first and second follow-up collected as dynamics are categorized from 0 to 4 (0: All symptoms have gone away; 1: Majority of symptoms has gone away; 2: Majority of symptoms is still present; 3: No changes in my symptoms; 4: Now I feel worse).

A dose of a new combination of agents, (Prolactis IVU ${ }^{\odot}$, Omega Pharma, Cantù, Italy) was administered twice daily for 5 days and then once a day for 10 days. According to the company data, Prolactis IVU contains D-mannose $2 \mathrm{~g}$, Prebiotics (fructo-oligosaccharide 1g), pomegranate extract $250 \mathrm{mg}$ (with 70\% titration of ellagic acid $175 \mathrm{mg}$ ) and Probiotics (Lactobacillus plantarum Lp1 $15 \geq 2$ billion colony-forming unit). Each component has a peculiar mechanism of action that contributes to contrast bacteria. D-mannose provides anti-adesive properties against bacteria, in particular E. coli (14). Pomegranate extract and, in particular ellagic acid, has proven anti flagellar-propelled motility of certain strains of E. coli (15).

Lactobacillus plantarum improves vaginal colonization of Lactobacilli, a natural mechanism of defense against urinary tract infections (16). In particular, Lactobacilli can prevent the adherence, growth and colonization of uropathogenic bacteria (17). Fructo-oligosaccharides are prebiotics, that stimulate the growth and activity of intestinal bacteria in the large bowel by acting as a substrate for them (18).

Together with the combination of agents, forced hydration (> 2 liters/day) has been strongly suggested. Patients were instructed to contact the Department in case of worsening, persistence or recurrence of symptoms.

In case a patient returned with ongoing complaints during the study period, further treatments, including antibiotics, were considered depending on the specific case and on physician choice.

Dynamics changes, changes in total ACSS and its subscales were assessed at day 15 (T1) via a telephone interview by a certified Urologist, and at day 30 (T2), during the end of study in-office visit.

Response to treatment in terms of complete response, improvement of symptoms, persistence of symptoms or worsening of symptoms were registered as efficacy out- come. Incidence of adverse events and numbers of secondary antibiotic treatment were also registered.

\section{Statistical analysis}

Descriptive statistics were performed calculating mean value and related standard deviation for continues variables as age, and by frequencies and percentages for categorical variables as menopausal status, presence of cystocele, diabetes mellitus, number of episodes per year $\geq 3$. Data regarding changes in dynamics were also reported as frequencies and percentages at first and second follow-up. Mean scores, mean differences between baseline and first and second follow-up along with their 95\% confidence interval were also reported. Paired t-test comparing mean scores between baseline and first or second follow-up visit was used in order to test the hypothesis that no differences exist after a period of administration of the studied compound in total or subscale scores. A p-value $<0,05$ was considered for statistical significance. All the analyses were performed using statistical package STATA 13.1

\section{Results}

Forty-two female patients were admitted at our institution, complaining of urinary symptom suggestive of UTI. Nine patients were excluded due to relevant comorbidities, neurologic bladder or grade IV cystocele according to Baden-Walker classification (19) with high post-void residual urine (> $100 \mathrm{ml})$.

Thirty-three patients were, eventually, enrolled (mean age $38 \pm 11.2$ ) and completed the treatment protocol. Twenty-one percent were postmenopausal, $24 \%$ had a clinically significant cystocele, $27 \%$ suffered from wellcompensated diabetes. About 79\% reported $<3$ episodes of UTI/year (Table 1).

At first follow-up (f-up) Tl visit, all symptoms or the

\section{Table 1.}

Characteristics of the study sample.

\begin{tabular}{|lc|}
\hline Characteristic & Study sample $(\mathbf{n}=\mathbf{3 3})$ \\
\hline Age (mean, SD) & $38.1(11.2)$ \\
\hline BMl $(n, \%)$ & $21(63.6)$ \\
$<25$ & $12(36.4)$ \\
$\geq 25$ & $7(21.2)$ \\
\hline Menopause $(n, \%)$ & $8(24.2)$ \\
\hline Cystocele $(n, \%)$ & $9(27.3)$ \\
\hline Diabetes $(n, \%)$ & \\
\hline Episodes per year $(n, \%)$ & $26(78.8)$ \\
$<3$ & $7(21.2)$ \\
\hline 3 & \\
\hline BMl: Body Mass Index. & \\
\hline
\end{tabular}

\section{Table 2.}

Dynamics at first and second follow-up visit ( $n^{\circ}$ and \%).

\begin{tabular}{|c|c|cc|}
\hline & & Follow-up 1 & Follow-up 2 \\
\hline \multirow{5}{*}{ Dynamic } & 0 & $2(6.1)$ & $19(57.6)$ \\
& 1 & $8(24.2)$ & $11(33.3)$ \\
& 2 & $16(48.5)$ & $3(9.1)$ \\
& 3 & $6(18.2)$ & - \\
& 4 & $1(3.0)$ & - \\
\hline
\end{tabular}




\begin{tabular}{|c|c|c|c|c|c|c|c|c|c|c|c|}
\hline & Total & \multicolumn{2}{|c|}{ BMI } & \multicolumn{2}{|c|}{ Menopause } & \multicolumn{2}{|c|}{ Cystocele } & \multicolumn{2}{|c|}{ Diabetes } & \multicolumn{2}{|c|}{ Episodes/year } \\
\hline \multicolumn{12}{|c|}{ Dynamic at first follow-up - $\mathbf{n}(\%)$} \\
\hline & & $<25$ & $\geq 25$ & Yes & No & Yes & No & Yes & No & $<3$ & $\geq 3$ \\
\hline$\overline{0}$ & $2(6.1)$ & $2(9.5)$ & $0(0)$ & $2(28.6)$ & $0(0)$ & $0(0)$ & $2(8.0)$ & 1 (11.1) & $1(4.17)$ & $2(7.7)$ & $0(0)$ \\
\hline 1 & $8(24.2)$ & $8(38.1)$ & $0(0)$ & $0(0)$ & $8(30.8)$ & $0(0)$ & $8(32.0)$ & $2(22.2)$ & $6(25.0)$ & $7(26.9)$ & $1(14.3)$ \\
\hline 2 & $16(48.5)$ & $9(42.9)$ & $7(58.3)$ & $2(28.6)$ & $14(53.9)$ & $5(62.5)$ & $11(44.0)$ & $2(22.2)$ & $14(58.3)$ & $12(46.1)$ & $4(57.1)$ \\
\hline 3 & $6(18.2)$ & $2(9.5)$ & $4(33.3)$ & $3(42.9)$ & $3(11.5)$ & $3(37.5)$ & $3(12.0)$ & $3(33.3)$ & $3(12.5)$ & $4(15.4)$ & $2(28.6)$ \\
\hline$\overline{4}$ & $1(3.0)$ & $0(0)$ & $1(8.3)$ & $0(0)$ & $1(3.9)$ & $0(0)$ & $1(4.0)$ & $1(11.1)$ & $0(0)$ & $1(3.9)$ & $0(0)$ \\
\hline$\overline{p \text { value }}$ & ie - & & & & & & & & & & \\
\hline \multicolumn{12}{|c|}{ Dynamic at second follow-up - $\mathrm{n}(\%)$} \\
\hline & & $<25$ & $\geq 25$ & Yes & No & Yes & No & Yes & No & $<3$ & $\geq 3$ \\
\hline$\overline{0}$ & $19(57.6)$ & $15(71.4)$ & $4(33.3)$ & $3(42.9)$ & $16(61.5)$ & $18(72.0)$ & $1(12.5)$ & $15(62.5)$ & $4(44.4)$ & $17(65.4)$ & $2(28.6)$ \\
\hline$\overline{1}$ & 11 (33.3) & $4(19.1)$ & $7(58.3)$ & $3(42.9)$ & $8(30.8)$ & $5(20.0)$ & $6(75.0)$ & $6(25.0)$ & $5(55.6)$ & $8(30.8)$ & $3(42.9)$ \\
\hline 2 & $3(9.1)$ & $2(9.5)$ & $1(8.3)$ & $1(14.3)$ & $2(7.7)$ & $2(8.0)$ & $1(12.5)$ & $3(12.5)$ & $0(0)$ & $1(3.9)$ & $2(28.6)$ \\
\hline 3 & - & - & - & - & - & - & - & - & - & - & - \\
\hline 4 & - & - & - & - & - & - & - & - & - & - & - \\
\hline$\overline{\mathrm{p} \text { value }}$ & - & & & & & & & & & & \\
\hline
\end{tabular}

Table 3.

Table 4.

\begin{tabular}{|c|c|c|c|c|}
\hline \multirow[b]{3}{*}{ ACSS Sub-scales } & \multicolumn{4}{|c|}{ Mean ( $95 \%$ Confidence Interval) } \\
\hline & \multicolumn{4}{|c|}{ ACSS scores changes between baseline and first follow-up } \\
\hline & Baseline & First Follow-up & $\begin{array}{l}\text { Difference between baseline } \\
\text { and first follow-up }\end{array}$ & $p$-value \\
\hline Typical & $11.5(10.5$ to 12.6$)$ & $4.9(4.0$ to 5.9$)$ & $-6.6(-5.9$ to -7.3$)$ & $<0.0001$ \\
\hline Differential & 3.1 (2.6 to 3.6$)$ & $0.6(0.3$ to 0.9$)$ & $-2.5(-2.0$ to -2.9$)$ & $<0.0001$ \\
\hline \multirow[t]{3}{*}{ Quality of Life } & $7.2(6.7$ to 7.7$)$ & $4.0(3.3$ to 4.6$)$ & $-3.2(-2.5$ to -3.9$)$ & $<0.0001$ \\
\hline & \multicolumn{4}{|c|}{ ACSS scores changes first and second follow-up } \\
\hline & First Follow-up & Second Follow-up & $\begin{array}{l}\text { Difference between first } \\
\text { and second follow-up }\end{array}$ & p-value \\
\hline Typical & $4.9(4.0$ to 5.9$)$ & 2.7 (2.1 to 3.3$)$ & $-2.2(-1.4$ to -3.0$)$ & $<0.0001$ \\
\hline Differential & $0.6(0.3$ to 0.9$)$ & $0.3(0.1$ to 0.5$)$ & $-0.3(-0.1$ to -0.6$)$ & 0.009 \\
\hline \multirow[t]{3}{*}{ Quality of Life } & $4.0(3.3$ to 4.6$)$ & $1.7(1.2$ to 2.1$)$ & $-2.3(-1.7$ to -2.8$)$ & $<0.0001$ \\
\hline & \multicolumn{4}{|c|}{ ACSS scores changes between baseline and second follow-up } \\
\hline & Baseline & Second Follow-up & $\begin{array}{l}\text { Difference between baseline } \\
\text { and second follow-up }\end{array}$ & $p$-value \\
\hline Typical & $11.5(10.5$ to 12.6$)$ & 2.7 (2.1 to 3.3$)$ & $-8.8(-7.8$ to -9.8$)$ & $<0.0001$ \\
\hline Differential & 3.1 (2.6 to 3.6$)$ & $0.3(0.1$ to 0.5$)$ & $-2.8(-2.2$ to -3.3$)$ & $<0.0001$ \\
\hline Quality of Life & $7.2(6.7$ to 7.7$)$ & 1.7 (1.2 to 2.1$)$ & $-5.5(-4.9$ to -6.1$)$ & $<0.0001$ \\
\hline
\end{tabular}

$\mathrm{T} 1$ and to $1.7(1.2-2.1)$ at $\mathrm{T} 2$ (p-values < $0.0001)$. Also difference in mean scores between baseline and $\mathrm{T} 1$ and $\mathrm{T} 2$ are reported in Table 4. Six patients required antibiotics, during T1 (2 patients after 5 days and 4 patients after 7 days) due to symptoms persistence or worsening. These patients were included in symptoms analysis too both in $\mathrm{Tl}$ and T2. No adverse events were recorded.

\section{Discussion}

The aim of this pilot study was to investigate a new combination of D-Mannose, pomegranate extract, prebiotics and probiotics as an effective treatment in modifying a specific questionnaire in women with acute uncomplicated cystitis. ACSS is an 18-item selfreporting questionnaire including six questions about "typical" symptoms of AUC, four questions regarding "differential" symptoms

majority of symptoms went off in 10 women (30.3\%) (Table 2 - Dynamics at T1) and at second follow-up visit in 30 women (90.9\%) (Table 3 - Dynamics at T2); some symptoms still remained in 16 women (48.5\%) at $\mathrm{T} 1$ and in 3 women (9.1\%) at T2. Persistence of symptoms or the worsening of the condition were observed in 7 patients $(21.2 \%)$ at $\mathrm{T} 1$ and in none at T2. BMI and premenopausal state were statistically associated with worse symptom score at $\mathrm{T} 1$ ( $\mathrm{p}=0.015$ for $\mathrm{BMI} \geq 25$ and $\mathrm{p}=$ 0.01 for pre-menopausal state). This difference was not confirmed at $\mathrm{T} 2$ whereas a trend of association was present for patients with cystocele $(p=0.06)$ (Table 2$)$.

Regarding the mean score at the ACSS subscales, the analysis showed a significant reduction in all the subscales between baseline visit and both T1 and T2. The reported mean score in typical symptoms at baseline was 11.5 (10-5--12-6), falling to $4.9(4.0-5.9)$ at $\mathrm{T} 1$ and to $2.7(2.1-3.3)$ at T2 (p-values $<0.0001)$. The mean score in differential symptoms was 3.1 (2.6-3.6) at baseline, falling to $0.6(0.3-0.9)$ at $\mathrm{T} 1$ and to $0.3(0.1-0.5)$ at $\mathrm{T} 2$ (p-values 0.009 to $<0.0001$ ). Lastly, the QoL mean score was $7.2(6.7-7.7)$ at baseline, falling to $4.0(3.3-4.6)$ at suggestive of alternative diagnoses and three questions on quality of life items (11-13, 20).

Even if antibiotic therapy is currently the treatment of choice due to high efficacy, this must be balanced against the emergence of microorganism resistant to a great variety of antibiotics. Thus, the pain and discomfort of the UTI must be balanced with the cost and risk of developing resistance when using antimicrobials (4-5, 21-23). Thus, even if continuous prophylaxis and self-starting antibiotic treatment are commonly accepted options for prophylaxis, a non-antibiotic management could be effective also in limiting these recurrences (24-28).

The results of this pilot study showed that the majority of patients enrolled were safely treated with progressive resolution of symptoms. In particular, after fifteen days all symptoms or the majority of symptoms went off in 10 women (30.3\%) and after thirty days this result was obtained in $90.9 \%$ of women.

Thus, our results sustain the hypothesis that UTI is a self-limiting disorder in many patients. Even without antibiotic treatment, symptomatic infection seems to heal in a substantial number of women (24). There is no 
general consensus in clinical trial results. A trial by Christiaens et al. compared clinically suspected UTI treated with nitrofurantoin or placebo. In the placebo group, more than half had some symptomatic improvement and the effect of the treatment was not statistically significant ( $\mathrm{p}=0.08)(29)$.

Conversely, Ferry et al. compared different pivmecillinam regimens and a placebo in a large UTI trial with slightly a clear advantage in favor of any antibiotic regimen in terms of symptoms resolution (30).

Moreover, another study of Ferry et al., which included 288 patients placebo-treated showed slow decline of symptom. After one week, 30\% of women were completely symptoms-free, while $75 \%$ were free from suprapubic and loin pain, 45\% from urgency and dysuria. At end of study (at six weeks) percentage rose up to 90, 70 and $55 \%$, respectively for specific symptoms and to 54\% for all symptoms (31). Our result at one-month follow-up is quite similar (57.6\%) of complete symptom resolution. To our knowledge, this is the first study which analyses in a real life setting whether hyperhydration and a formula of D-mannose, prebiotics, probiotics and pomegranate extract might be effective in modifying a cystitisspecific questionnaire in women with symptoms of acute cystitis. However, none of these studies involved a symptomatic treatment arm. There are some limitations in our study. Lack of control arm and microbiological confirmation of UTI are the main limitations. However, in common clinical practice it is difficult to perform a urine culture in many situations like in the week-end or in the evening and night.

Moreover, we included some women who do not fit the definition of uncomplicated UTI according to EAU guidelines, including also post-menopausal women, diabetic and with pelvic organ prolapse. A strength point could be that we included also a subset of recurrent UTIs (rUTIs). In fact rUTIs that are defined as having greater than two infections in a 6-month period, or three infections over twelve months, are included in uncomplicated UTI but are typically excluded in randomized clinical trial dealing with AUC. Another limitation of the study is the absence of long term follow up to investigate recurrence in the months after the treatment. Given the uncontrolled design of the study, it is not possible to verify the amount of contribution of each component of the formula.

\section{Conclusions}

Our study suggests that the synergistic action of the components of this new combination could help the bacterial washout resulting in an effective management of acute cystitis in women without antibiotics in a wide majority of the cases. This approach was also safe because no women had consequences of non-antibiotic management. However, a larger sample size and longer follow-up are needed to confirm these promising results.

\section{REFERENCES}

1. Flores-Mireles AL, Walker JN, Caparon M, Hultgren SJ. Urinary tract infections: epidemiology, mechanisms of infection and treatment options. Nat Rev Microbiol. 2015; 13:269-84.
2. Bonkat G, Bartoletti RR, Bruyère F, et al. EAU Guidelines on Urological Infections. Retrieved from:https://uroweb.org/guideline/ urological-infections/Access date 05/08/2019.

3. Bent S, Nallamothu BK, Simel DL, et al. Does this woman have an acute uncomplicated urinary tract infection? JAMA. 2002; 287:2701-2710.

4. Chen $Y H, K o W C$, Hsueh PR. Emerging resistance problems and future perspectives in pharmacotherapy for complicated urinary tract infections. Expert Opin. Pharmacother. 2013; 14:587-596.

5. Bader MS, Loeb M, Brooks AA. An update on the management of urinary tract infections in the era of antimicrobial resistance. Postgrad Med. 2017; 129:242-258.

6. Schwenger EM, Tejani AM, Loewen PS. Probiotics for preventing urinary tract infections in adults and children. Cochrane Database Syst Rev, 2015; (12):CD008772.

7. Stothers L. A randomized trial to evaluate effectiveness and cost effectiveness of naturopathic cranberry products as prophylaxis against urinary tract infection in women. Can J Urol. 2002; 9:1558-62.

8. Kranjcec B, Papeš D, Altarac S. D-mannose powder for prophylaxis of recurrent urinary tract infections in women: a randomized clinical trial. World J Urol. 2014; 32:79-84.

9. Beerepoot MA, Geerlings SE, van Haarst EP, et al. Nonantibiotic prophylaxis for recurrent urinary tract infections: a systematic review and meta-analysis of randomized controlled trials. J Urol 2013; 190:1981-89.

10. World Medical Association. World Medical Association Declaration of Helsinki: ethical principles for medical research involving human subjects. JAMA 2013; 310:2191-4.

11. Alidjanov JF, Abdufattaev UA, Makhsudov SA, et al. New selfreporting questionnaire to assess urinary tract infections and differential diagnosis: acute cystitis symptom score. Urol Int. 2014; 92:230-36.

12. Alidjanov JF, Naber KG, Abdufattaev UA, et al. Reliability of Symptom-Based Diagnosis of Uncomplicated Cystitis. Urol Int. 2019; 102:83-95.

13. Alidjanov JF, Naber KG, Abdufattaev UA, et al. Reevaluation of the Acute Cystitis Symptom Score, a self-reporting suestionnaire. Part I. Development, diagnosis and differential diagnosis. Antibiotics (Baseel) 2018; 7(1). pii: E6.

14. Wellens A, Garofalo C, Nguyen H, et al. Intervening with urinary tract infections using anti-adhesives based on the crystal structure of the FimH-oligomannose-3 complex. PLoS One. 2008; 3:e2040.

15. Asadishad B, Hidalgo G, Tufenkji N. Pomegranate materials inhibit flagellin gene expression and flagellar-propelled motility of uropathogenic Escherichia coli strain CFT073. FEMS Microbiol Lett. 2012; 334:87-94.

16. Vladareanu R, Mihu D, Mitran M, et al. New evidence on oral L. plantarum $P 17630$ product in women with history of recurrent vulvovaginal candidiasis (RVVC): a randomized double-blind placebo-controlled study. Eur Rev Med Pharmacol Sci. 2018; 22:262267.

17. Akgül T, Karakan T. The role of probiotics in women with recurrent urinary tract infections. Turk J Urol. 2018; 44:377-383.

18. Liao N, Luo B, Gao J, et al. Oligosaccharides as co-encapsulating agents: effect on oral Lactobacillus fermentum survival in a simulated gastrointestinal tract. Biotechnol Lett. 2019; 41:263-272.

19. BadenWF, WalkerTA. Genesis of the vaginal profile: a correlat- 
ed classification of vaginal relaxation.Clin Obstet Gynecol. 1972; 15:1048-54.

20. Alidjanov JF, Naber KG, Pilatz A, et al. Evaluation of the draft guidelines proposed by EMA and FDA for the clinical diagnosis of acute uncomplicated cystitis in women. World J Urol. 2020; 38:63-72.

21. Pendleton JN, Gorman SP, Gilmore BF. Clinical relevance of the ESKAPE pathogens. Expert Rev. Anti Infect Ther. 2013; 11:297308.

22. Bauer KA, Kullar R, Gilchrist M, File TM Jr. Antibiotics and adverse events: the role of antimicrobial stewardship programs in 'doing no harm'. Curr Opin Infect Dis. 2019; 32:553-558.

23. Fenwick EA, Briggs AH, Hawke CI. Management of urinary tract infection in general practice: a cost-effectiveness analysis. $\mathrm{Br} \mathrm{J}$ Gen Pract. 2000; 50:635-9.

24. Sihra N, Goodman A, Zakri R, et al. Nonantibiotic prevention and management of recurrent urinary tract infection. Nat Rev Urol. 2018; 15:750-776.

25. Bleidorn J, Gagyor I, Kochen MM, et al. Symptomatic treatment (ibuprofen) or antibiotics (ciprofloxacin) for uncomplicated urinary tract infection? Results of a randomized controlled pilot trial. BMC Med. 2010; 8:30.

26. Wagenlehner FM, Vahlensieck W, Bauer HW, et al. Prevention of recurrent urinary tract infections. Minerva Urol Nefrol. 2013; 65:9.

27. Del Popolo G, Nelli F. Recurrent bacterial symptomaticcystitis: A pilot study on a new natural option for treatment. Arch Ital Urol Androl. 2018; 90:101-103.

28. Vicariotto F. Effectiveness of an association of a cranberry dry extract, D-mannose, and the two microorganismsLactobacillus plantarum LP01 and Lactobacillusparacasei LPC09 in women affected by cystitis: a pilot study. J Clin Gastroenterol. 2014; 48 Suppl 1:S96-101.

29. Christiaens TC, De Meyere M, Verschraegen G, et al. Randomised controlled trial of nitrofurantoin versus placebo in the treatment of uncomplicated urinary tract infection in adult women. Br J Gen Pract. 2002; 52:729-34.

30. Ferry SA, Holm SE, Stenlund H, et al. Clinical and bacteriological outcome of different doses and duration of pivmecillinam compared with placebo therapy of uncomplicated lower urinary tract infection in women: the LUTIW project. Scand J Prim Health Care. 2007; 25:49-57.

31. Ferry SA, Holm SE, Stenlund H, et al. The natural course of uncomplicated lower urinary tract infection in women illustrated by a randomized placebo controlled study. Scand J Infect Dis. 2004; 36:296-301.

\section{Correspondence}

Dario Pugliese, MD (Corresponding Author) dariopugliese87@gmail.com

Luca Cindolo, MD

lucacindolo@gmail.com

Department of Urology "Villa Stuart" Private Hospital

Via Trionfale, 5952 Rome (Italy)

Anna Acampora, MD

anna.acampora@unicatt.it

Institute of Public Health, Catholic University of Sacred Heart

Largo A. Gemelli, 8 Rome (Italy)

Angelo Porreca, MD

angelo.porreca@casacura.it

Department of Robotic Urological Surgery, Abano Terme Hospital

Piazza Cristoforo Colombo, 1, 35031 Abano Terme PD (Italy)

Luigi Schips, MD

luigischips@hotmail.com

Department of Urology, "G. D'Annunzio" University

Via S. Camillo de Lellis, 66054 Vasto (CH) (Italy) 